\title{
Développement d'un vaccin contre Haemophilus influenzae de sérotype a : Compte-rendu d'un atelier
}

\author{
AD Cox $^{1 *}$, L Barreto ${ }^{1}$, M Ulanova², MG Bruce ${ }^{3}$ et RSW Tsang ${ }^{4}$ au nom des collaborateurs à la \\ conférence ${ }^{5}$
}

\section{Résumé}

Depuis la fin des années 1990, nous observons une émergence d'infections causées par Haemophilus influenzae de sérotype a (Hia), en particulier dans les communautés autochtones situées dans le nord du Canada et de l'Alaska, et ces infections sont associées à une importante morbidité et à un taux de mortalité d'environ $10 \%$. Un vaccin contre Hia pourrait peut-être prévenir cette maladie et permettre au système de soins de santé de réaliser des économies de plusieurs millions de dollars dans le domaine des soins actifs et des soins de longue durée.

Les 23 et 24 mars 2016, le Conseil national de recherches du Canada (CNRC), I'Agence de la santé publique du Canada (ASPC) et les Instituts de recherche en santé du Canada (IRSC) ont tenu une rencontre conjointe sur $\mathrm{H}$. influenzae de sérotype a (Hia), afin d'examiner l'état actuel de l'épidémiologie de la maladie et de discuter du développement possible d'un vaccin. Cette rencontre a réuni des représentants du milieu universitaire, de bureaux de santé publique fédéral et territoriaux, de laboratoires d'hôpitaux, de ministères fédéraux concernés par la santé des Autochtones, d'organismes de défense des droits des peuples autochtones et de l'industrie.

Les représentants de l'industrie ont confirmé que l'industrie était intéressée à appuyer la préparation de lots de vaccins à des fins d'essais cliniques et qu'elle avait la capacité nécessaire à cette fin. Les organismes de réglementation canadiens se sont dits pour leur part disposés à assurer la mise en place des mesures appropriées la license du produit. II existe en outre une capacité et un intérêt sur la conduite d'essais cliniques dans certaines communautés autochtones du Canada et de l'Alaska. Diverses recommandations ont été formulées en ce qui a trait aux prochaines étapes, notamment les suivantes : terminer les essais précliniques; améliorer la surveillance épidémiologique afin de mieux comprendre l'étendue de la maladie dans le reste de l'Amérique du Nord et à l'échelle mondiale; mettre en place des mécanismes d'engagement avec les organismes autochtones nationaux pour assurer la pleine participation de leurs peuples au processus et examiner les possibilités de financement en vue de la production de lots pour les essais cliniques et de la conduite de ces essais.

\author{
Affiliations \\ ${ }^{1}$ Portefeuille des Thérapeutiques \\ en santé humaine, Conseil \\ national de recherches du \\ Canada, Ottawa (Ontario) \\ ${ }^{2}$ École de médecine du Nord de \\ I'Ontario, Université Lakehead, \\ Thunder Bay (Ontario)
}

${ }^{3}$ Arctic Investigation Program, Division of Preparedness and Emerging Infections, United States Centers for Disease Control and Prevention, Anchorage (Alaska)

${ }^{4}$ Laboratoire national de microbiologie, Agence de la santé publique du Canada, Winnipeg (Manitoba)

${ }^{5}$ Tous les collaborateurs à la conférence sont mentionnés à la fin du document

*Correspondance : andrew.cox@
nrc-cnrc.gc.ca

Citation proposée : Cox AD, Barreto L, Ulanova M, Bruce MG, Tsang RSW au nom des collaborateurs à la conférence. Développement d'un vaccin contre Haemophilus in luenzae de stype a : Compte-rendu d'un atelier. Relevé des maladies transmissibles au Canada. 2017;43(5):98-105. https://doi.org/10.14745/ccdr.v43i05a02f

\section{Introduction}

Haemophilus influenzae est une bactérie Gram négative qui peut causer un éventail varié d'infections allant de l'otite moyenne à la septicémie. Les souches de $H$. influenzae se divisent entre les souches qui présentent en surface des capsules polysaccharidiques qui, sur le plan antigénique, répartissent les souches en six sérotypes capsulaires différents (de a à f) et les souches sans capsule (dont le type ne peut être déterminé). $H$. influenzae de sérotype $b(\mathrm{Hib})$ était la cause la plus fréquente de méningite chez les enfants jusqu'à ce qu'un vaccin glycoconjugué basé sur la capsule polysaccharidique de Hib soit intégré au programme systématique de vaccination des nourrissons au Canada et aux États-Unis, au début des années 1990 (1). Le vaccin contre Hib a été très efficace pour réduire les infections causées par Hib; depuis la fin des années 1990, toutefois, un nombre croissant d'infections causées par
$H$. influenzae de sérotype a (Hia) ont été recensées, notamment dans les communautés autochtones des régions du nord du Canada et de l'Alaska (2). Or, les infections causées par Hia peuvent être graves. Une récente étude menée auprès de jeunes enfants a ainsi révélé que $42 \%$ étaient atteint d'une méningite, $19 \%$ d'une pneumonie bactériémique, $25 \%$ d'une infection des os, des articulations et des tissus mous et $11 \%$ en sont décédés (3). II n'existe à l'heure actuelle aucun vaccin.

Les 23 et 24 mars 2016, le Conseil national de recherches du Canada (CNRC), l'Agence de la santé publique du Canada (ASPC) et les Instituts de recherche en santé du Canada (IRSC) ont tenu une rencontre conjointe sur Hia, afin d'examiner l'état actuel de l'épidémiologie de la maladie et de discuter du développement possible d'un vaccin. Cette rencontre a réuni 
des représentants du milieu universitaire, de bureaux de la santé publique fédérale et territoriale, de laboratoires d'hôpitaux, de ministères fédéraux concernés par la santé des Autochtones, d'organismes de défense des droits des peuples autochtones et de l'industrie.

Le présent article résume les renseignements communiqués durant cette rencontre, notamment les récentes tendances relatives à l'épidémiologie du Hia au Canada et ailleurs, examine la manière dont les peuples autochtones participent, et peuvent participer, au développement d'un vaccin contre Hia, résume I'immunologie de Hia et les recherches précliniques sur le vaccin contre Hia, examine diverses options en vue de la mise au point d'un vaccin et formule des recommandations sur les prochaines étapes à entreprendre.

\section{L'épidémiologie de Haemophilus influenzae de sérotype a}

\section{Hia à l'échelle mondiale : Une vue d'ensemble $D^{r}$ Raymond Tsang}

Une étude a été effectuée le 4 mars 2016 sur le site Web Typage génomique multilocus de $H$. influenzae (4) pour évaluer la présence du Hia à l'échelle mondiale. L'examen de cet ensemble de données a révélé la présence de Hia en Amérique du Nord et en Amérique du Sud, en Afrique, en Europe, en Asie et en Australie. Les premiers rapports sur l'infection causées par Hia remontent à 1980 et portent sur des cas recensés en Gambie, en Papouasie-Nouvelle-Guinée et en République dominicaine, ce qui témoigne d'une distribution mondiale du pathogène. Le site Web sur le Typage génomique multilocus faisait également mention d'une grappe de cas d'infection invasive à Hia observée au Nouveau-Mexique en 2009-2010, qui s'est révélée être causée par une souche de Hia présentant le même type de séquence que la souche observée en Alaska (5).

Des données semblent indiquer une augmentation de l'incidence du Hia dans certaines régions depuis l'introduction du vaccin contre Hib. À titre d'exemple, selon une étude représentative de la population menée de 1998 à 2008 au Utah auprès d'enfants âgés de moins de 18 ans (6), le taux d'incidence d'infections causées par Hia a augmenté, passant de 0,8 pour 100000 à 2,6 pour 100000 . Une étude comparable a révélé que le taux d'incidence d'infections invasives causées par Hia était de 2,7 pour 100000 chez les personnes âgées de 65 ans et plus (7). Au Brésil, avant l'introduction du vaccin conjugué contre Hib, 97,8\% de toutes les infections invasives dues à $H$. influenzae étaient attribuables au sérotype b, le sérotype a (Hia) ne représentant que $0,5 \%$ des cas (8). Cinq ans après I'introduction du vaccin conjugué contre Hib, l'incidence globale de la méningite causées par Hib dans la population générale avait diminué de 2,39 à 0,06 pour 100000 habitants. Durant cette periode, il a été observé que Hia (deux clones) a remplacé graduellement ce sérotype (9). L'évolution de l'épidémiologie de l'infection invasive causées par $H$. influenzae à l'échelle mondiale mérite donc d'être examinée plus à fond.

\section{Hia au Canada : Une étude de série de cas}

Dr David Scheifele

Le Programme canadien de surveillance active de l'immunisation (IMPACT) est un réseau national de surveillance active en milieu hospitalier auquel participent 12 hôpitaux pédiatriques du Canada. Ces hôpitaux représentent plus de 90 \% des lits pédiatriques de soins tertiaires au Canada $(10,11)$. Une surveillance des infections invasives causées par $H$. influenzae (tous sérotypes confondus) est exercée depuis 2007. Les cas sont définis à partir d'un isolat prélevé d'un site normalement stérile et confirmés comme appartenant au sérotype a (Hia) par le Laboratoire national de microbiologie (LNM) de l'Agence de la santé publique du Canada (ASPC) de Winnipeg. Les cas observés proviennent à la fois des provinces et des territoires.

Au total, 102 cas d'infection causées par Hia ont été recensés depuis 2007, soit en moyenne 12,5 cas par année, le tiers d'entre eux provenant des territoires. La plupart des cas ont été observés à Winnipeg, à Edmonton et à Montréal, qui servent de centres de référence pour les territoires. À partir du code postal lié à chaque cas d'origine provinciale, il a été possible de déterminer que les infections étaient réparties sur un vaste territoire géographique et, surtout, que ces infections ne constituaient pas un risque uniquement dans le Grand Nord. II est également apparu clairement que plus de la moitié des cas pédiatriques s'étaient manifestés chez des nourrissons de moins d'un an, certains étant à peine âgés de quelques semaines. Près des deux tiers des cas ont été observés chez des enfants de moins de deux ans. L'infection était souvent grave et nécessite des soins intensifs; neuf pour cent des personnes atteintes sont décédées des suites de l'infection. Presque tous les cas touchaient des Autochtones. En termes de gravité, la moitié des personnes infectées ont contracté la méningite, pneumonie, otite moyenne et l'arthrite purulente étant également répandues. Enfin, des crises d'épilepsie ont été observées chez plus de la moitié des personnes atteintes de méningite, cela témoignant d'une infection à un stade avancé au moment du diagnostic.

\section{Hia au Québec}

$D^{\text {re }}$ Andrée-Anne Boisvert

Les infections invasives causées par Hia constituent un grave problème de santé chez les enfants autochtones vivant dans le nord du Québec, notamment chez les Cris du Nunavik et de la baie James où ont été observés 40 des 62 cas recensés de 2006 à 2015 (2). La majorité des cas touchent des jeunes enfants, $66 \%$ d'entre eux ayant moins de cinq ans. Cela équivaut à des sommets d'environ 100 cas pour 100000 habitants de tous âges, de 225 pour 100000 enfants de moins de cinq ans et de 350 pour 100000 enfants de moins d'un an. Dans le territoire cri de la baie James, ces chiffres sont plus élevés que les taux d'incidence des infections causées par Hib avant l'instauration de la vaccination. Le taux de mortalité est de $15 \%$ et tous les décès sont survenus dans le peuple inuit. Presque tous les cas exigent une évacuation par hélicoptère (Medi-Vac) vers un centre urbain. 


\section{Hia au Nunavut}

Dre Kim Barker

Le Nunavut compte 32000 habitants, dont $90 \%$ sont des Inuits. Le Nunavut a mis en place un programme de vaccination contre le Hib financé par l'État. Depuis 2007, la majorité des infections invasives causée par $\mathrm{H}$. influenzae l'ont été par le sérotype a (plus de $60 \%$ des cas) et plus de $90 \%$ des infections causée par Hia ont été observées chez des enfants de moins de deux ans, l'incidence étant par ailleurs légèrement plus élevée chez les sujets de sexe masculin (3). Dans l'ensemble, les taux d'incidence des infections causée par Hia sont comparables dans les différentes régions du Nunavut, à l'exception de des sommets observés certaines années dans la région de Kitikmeot. Ces sommets correspondent à des taux de 140 à 160 cas pour 100000 habitants.

La gravité de la maladie progresse rapidement. En raison du caractère éloigné de certaines régions et de la rapidité de progression de la maladie, les régions dépendent entièrement de l'évacuation médicale par hélicoptère (Medi-Vac) pour le traitement de chaque enfant atteint d'une infection invasive à Hia. Comme on compte en moyenne six cas par année et qu'il en coûte 55000 \$ par évacuation, les frais de transport liés aux infections à Hia totalisent à eux seuls environ 0,33 million de dollars par année.

\section{Hia en Alaska}

Dr Michael Bruce

Depuis 2000, plus de 50 cas d'infection invasive causée par Hia ont été signalés en Alaska (5) (et données inédites de M.G.B.), ce qui représente $60 \%$ de toutes les infections causée par $H$. influenzae recensées dans cet État. Quatre-vingt-cinq pour cent de ces cas étaient concentrés dans l'ouest de l'État. Les infections touchent en très grande majorité les très jeunes enfants, $33 \%$ ayant moins de sept mois, $67 \%$ moins d'un an et $83 \%$ moins de deux ans. L'âge moyen des sujets atteints d'une infection invasive causée par Hia est de huit mois; 67 \% d'entre eux sont de sexe masculin et $92 \%$ sont d'origine autochtone. Cela équivaut à des taux d'incidence annuels de 13 pour 100000 chez les enfants de moins de deux ans et de 44 pour 100000 chez les Alaskiens d'origine âgés de moins de deux ans. Plus de $90 \%$ des personnes atteintes d'une infection invasive causée par Hia ont été vaccinées contre le Hib à l'âge prévu et plus de $80 \%$ ont dû être hospitalisées; le taux de létalité est de $8 \%$. Comme ces personnes vivent dans une région éloignée, $80 \%$ d'entre elles ont nécessité un transport aérien. Au cours de la dernière décennie, peu de variations ont été observées dans les trois types de séquence (ST 576, ST 23 et ST 56), bien que le ST 56 soit dominant depuis peu (5). Le choix du vecteur protéique a également été déterminant dans le succès du vaccin contre Hib en Alaska, le remplacement de l'anatoxine diphtérique par le complexe protéique de la membrane externe de Neisseria meningitidis comme vecteur protéique s'étant révélé nécessaire pour obtenir des titres protecteurs (12); le remplacement subséquent du complexe protéique de la membrane externe par une variante génétique de l'anatoxine diphtérique comme vecteur a toutefois favorisé la réapparition de l'infection causée par Hib, une situation qui n'a pu être corrigée que par un retour à l'utilisation du complexe protéique de la membrane externe comme vecteur protéique $(13,14)$.

\section{Participation des peuples autochtones au développement d'un vaccin contre Hia}

La mobilisation précoce des peuples autochtones est absolument vitale pour s'assurer que les communautés qui sont actuellement les plus menacées par ce nouveau pathogène soient mises à contribution et soient conscientes de la menace et de la solution possible.

\section{Les IRSC et l'approche du " double regard " pour la recherche sur les Premières Nations, les Inuits et les Métis}

D rimon Brascoupé

Les priorités des Instituts de recherche en santé du Canada incluent l'amélioration des expériences et des résultats pour les patients grâce à l'innovation en santé, la santé et le bien-être des peuples autochtones, l'amélioration des perspectives en santé par l'adoption de mesures de prévention, ainsi que l'amélioration de la qualité de vie des personnes atteintes d'affections chroniques. Ces priorités doivent être envisagées parallèlement avec la vision stratégique de l'Institut de la santé des Autochtones (ISA) qui s'énonce comme suit : Les Premières Nations, les Inuits et les Métis et leurs communautés : forces vives de la recherche en santé et de l'application des connaissances pour ces populations, afin de transformer la santé des peuples autochtones par les systèmes de connaissances autochtones et la promotion du bien-être, de la force et de la résilience des peuples autochtones.

II ne fait aucun doute que ces priorités cadrent parfaitement avec la mise au point d'un vaccin glycoconjugué contre Hia. Il est toutefois essentiel de s'assurer que ce développement se fasse en collaboration avec les peuples autochtones, en tenant compte de la manière dont ces peuples perçoivent la santé et le bien-être. Pour y parvenir, toutefois, nous devons modifier notre approche en matière de recherche, afin de favoriser la recherche menée par les peuples autochtones et en collaboration avec eux, plutôt que la recherche sur les peuples autochtones. Il est également essentiel d'adopter une approche axée sur le "double regard ", c'est-à-dire une approche qui allie les perspectives occidentales et autochtones, afin de créer une nouvelle norme d'excellence en matière de recherche sur les Premières Nations, les Inuits et les Métis qui augmente la pertinence des priorités de recherche communautaire ainsi que des connaissances, des valeurs et des cultures des Premières Nations, des Inuits et des Métis. 


\section{Principes d'engagement de l'Assemblée des Premières Nations}

$D^{\text {re }}$ Marlene Larocque

L'Assemblée des Premières Nations (APN) est une instance politique qui appuie la santé des Premières Nations. Elle recueille de l'information avec les différents partenaires en vue d'améliorer les résultats pour les Premières Nations. Les principes d'engagement visant à favoriser les partenariats avec I'APN incluent les suivants : les politiques et les programmes qui sont élaborés de concert avec les Premières Nations donnent de meilleurs résultats et, plus cette collaboration s'amorce tôt, meilleurs sont les résultats; les lignes directrices des IRSC pour la recherche en santé chez les peuples autochtones sont une bonne ressource, et il importe d'adopter une approche holistique pour protéger les connaissances autochtones et de tenir compte des pratiques autochtones pour assurer la sécurité des enfants. Il est également essentiel de reconnaître que le fardeau de la maladie peut être influencé par l'environnement physique, notamment par le surpeuplement des logements et le manque d'accès à de I'eau courante potable.

\section{Direction générale de la santé des Premières nations et des Inuits de Santé Canada}

Kathleen Lydon-Hassan

La Direction générale de la santé des Premières nations et des Inuits (DGSPNI) de Santé Canada collabore avec ses partenaires en vue d'améliorer les résultats en matière de santé, de donner accès à des services de santé de qualité et de permettre aux Premières Nations et aux Inuits d'exercer un plus grand contrôle sur le système de santé. L'objectif global est d'amener le gouvernement fédéral, provinciaux et territoriaux, les Premières Nations et les Inuits à s'engager sur une voie commune axée sur I'amélioration de la santé. La DGSPNI appuie les programmes de vaccination dans les réserves, en assumant directement l'exécution de ces programmes ou en offrant une aide financière à des communautés ou organismes des Premières Nations vivant dans les réserves pour la prestation de ces programmes.

\section{Immunité contre le Hia et recherche préclinique sur le vaccin contre Hia}

\section{Immunité naturelle acquise contre le Hia \\ Dre Marina Ulanova}

Des chercheurs de l'École de médecine du Nord de l'Ontario travaillent avec diligence en vue d'établir des liens avec les communautés des Premières Nations et collaborent étroitement avec le Centre de santé Meno Ya Win de Sioux Lookout afin d'examiner la spécificité de l'immunité acquise naturellement contre le Hia. Le nord de l'Ontario est une région où l'incidence des infections invasives est élevée. De fait, de 2002 à 2016, les taux d'incidence des infections causée par Hia chez les enfants de moins de cinq ans ont varié de 7,7 à 23,2 pour 100000 $(15,16)$.
Curieusement, l'activité des anticorps bactéricides acquis naturellement contre Hia est plus élevée chez les adultes autochtones que chez les Non-autochtones, les titres géométriques moyens étant de 351 contre 183 respectivement, les Immunoglobuline $M(\lg M)$ étant plus répandus que les Immunoglobuline $\mathrm{G}(\mathrm{lgG})$ dans les anticorps spécifiques dirigés contre le polysaccharide capsulaire de Hia (17). Cependant, cette étude a aussi clairement démontré que les adultes en santé, tant autochtones que non autochtones, présentent des titres élevés d'anticorps bactéricides contre Hia, ce qui signifie que cette bactérie est présente dans l'ensemble de la communauté, et pas seulement chez les Autochtones (17).

Autre élément extrêmement important, il a été clairement démontré que l'activité bactéricide sérique acquise de façon naturelle contre Hia est presque entièrement attribuable aux anticorps dirigés contre les polysaccharides capsulaires. L'activité bactéricide a été facilement éliminée par absorbance avec des polysaccharides capsulaires purifiés de Hia. L'absorbance de sérums avec un polysaccharide de Streptococcus pneumoniae de sérotype 6B affichant une réactivité antigénique croisée n'a eu aucun effet sur les titres bactéricides contre Hia, ce qui souligne l'importance de la spécificité pour conférer une protection et appuie clairement le développement d'un vaccin glycoconjugué basé sur le polysaccharide capsulaire de Hia.

\section{Recherche sur le développement d'un vaccin contre Hia}

$D^{r}$ Andrew Cox

Des essais précliniques de recherche et développement ont été menés dans les laboratoires du Conseil national de recherches du Canada. Ces travaux ont mené à d'importantes réalisations, dont les suivantes:

- Croissance de plusieurs souches de Hia dans un milieu de culture non bovin approuvé, dans des fermenteurs de $30 \mathrm{~L}$, et l'établissement d'une soouche bactérienne de départ et d'une banque de cellules de deux souches (ASPC).

- Rendement de $400 \mathrm{mg} / \mathrm{L}$ de polysaccharides capsulaires pour Hia.

- Confirmation de la pureté des polysaccharides capsulaires isolés par spectroscopie de résonance magnétique nucléaire.

- Utilisation de méthodes de sonication pour optimiser la taille des polysaccharides capsulaires en préparation à la conjugaison.

- Oxydation des polysaccharides capsulaires de taille optimisée et détermination du degré d'oxydation requis par spectroscopie de résonance magnétique nucléaire.

- Optimisation de la conjugaison par amination réductrice directe avec plusieurs vecteurs protéiques, dont la sérum-albumine humaine, la protéine variante génétique de I'anatoxine dipthérique et la protéine $D$.

- Détermination de l'immunogénicité des vaccins conjugués à l'aide de l'essai immuno-enzymatique (ELISA), après vaccination de souris et de lapins.

- Détermination de l'activité fonctionnelle des antisérums ainsi obtenus : les meilleurs titres pour Hia ont été obtenus avec la protéine variante génétique de l'anatoxine dipthérique utilisée comme vecteur protéique lors des dosages bactéricides sériques, il ya une corrélation déjà établie avec les tites obtenus pour Hib. 
En plus de ces réalisations, aucune réactivité croisée sur le plan de la reconnaissance ou de la fonctionnalité n'a été observée entre les sérums de Hia et les souches de Hib, ou vice versa, ce qui témoigne de la spécificité de la réponse et de l'importance de développer un vaccin contre Hia qui est spécifique. La validation de principe a été établie, toutes les souches de Hia examinées ayant été détruites par le sérum dérivé du vaccin conjugué.

Les études futures porteront sur l'établissement de sérums fonctionnels avec des adjuvants acceptables (p. ex. alun) et sur l'examen des niveaux d'immunogénicité obtenus avec $d$ 'autres vecteurs protéiques.

\section{Facteurs à considérer dans le développement $d$ 'un vaccin}

Un grand nombre de facteurs importants doivent être pris en compte avant de passer du stade préclinique aux étapes suivantes du développement d'un vaccin. Parmi ces facteurs, mentionnons la capacité de produire un produit conforme aux bonnes pratiques de fabrication pour les essais cliniques, la prise en compte de toutes les exigences réglementaires (relativement à l'assurance de la qualité ou à la taille des essais cliniques) et la mise en place des infrastructures nécessaires à la conduite des essais cliniques.

\section{Capacités en matière d'essais cliniques au Canada}

\section{Dr Scott Halperin}

Le programme de recherche sur les vaccins au Canada est appuyé et surveillé par le programme IMPACT $(10,11)$, I'Association canadienne pour la recherche et l'évaluation en immunisation (CAIRE) (18) et le Réseau canadien de recherche sur l'immunisation (19). Le rôle du programme IMPACT est d'établir le fardeau d'une maladie, d'évaluer l'efficacité des vaccins et d'assurer la surveillance de la sécurité des vaccins. Le rôle de CAIRE est de favoriser et d'approfondir la recherche appliquée en vaccinologie afin que la population canadienne ait un accès rapide à des vaccins, nouveaux ou améliorés. Le Réseau canadien de recherche sur l'immunisation est un réseau multidisciplinaire de recherche qui réunit une centaine de chercheurs répartis dans plus de 35 centres du Canada et qui a l'appui de I'ASPC et des IRSC. II se concentre sur les derniers stades du cycle de vie d'un vaccin, de la sécurité à la programmation. Il procure une infrastructure officielle de recherche et de collaboration et ses objectifs principaux sont d'évaluer la sécurité et l'efficacité des vaccins et de maintenir une capacité d'intervention rapide.

Le Réseau canadien de recherche sur l'immunisation exploite un réseau d'essais cliniques par l'entremise d'un réseau principal de sites d'essais cliniques répartis dans l'ensemble du Canada. Ce réseau peut mener rapidement des essais auprès de vastes groupes et de groupes spécialisés, notamment des populations pédiatriques et des populations à risque, et a déjà mené des essais auprès des Premières Nations et des Métis (20).
Le Réseau canadien de recherche sur l'immunisationpourrait contribuer aux volets suivants du développement d'un vaccin contre Hia : études sur le fardeau de la maladie; essais cliniques de phase I et de phase II, études de modélisation et études coût-efficacité (MODERN), études sur les connaissances, les attitudes, les croyances et les comportements et évaluation des programmes.

\section{Aspects réglementaires}

\section{Dr Richard Siggers}

Depuis le stade préclinique de recherche et de développement jusqu'à la période d'autorisation après la mise en marché, une connaissance des exigences réglementaires favorisera l'efficacité du développement et augmentera la confiance dans la sécurité et la qualité des produits commercialisés. II est recommandé d'engager des consultations directes avec les organismes nationaux de réglementation à chacune des étapes du développement des produits. Il est notamment essentiel $\mathrm{d}^{\prime}$ adopter une approche utile en matière de caractérisation du produit pour assurer le succès de la fabrication des produits biologiques. La caractérisation des produits vise à déterminer les principaux critères de qualité des produits qui présentent un profil de sécurité approprié. Les critères de qualité se révèlent efficaces lors des essais cliniques. Ils serviront ensuite de mesures pour évaluer l'uniformité des produits après leur homologation afin de s'assurer que les produits fabriqués à l'échelle commerciale sont représentatifs du produit qui a été testé avec succès lors des essais cliniques. L'investissement de ressources dans la caractérisation du produit à un stade précoce du développement permettra de s'assurer que la transition, d'une fabrication à une échelle préindustrielle à une production à une échelle commerciale, n'aura pas d'incidence négative sur la qualité et la sécurité du produit.

La fabrication de produits biologiques (c.-à-d. les vaccins) est complexe, en raison de la variabilité et de la diversité inhérentes des produits de départ, de la complexité des systèmes biologiques utilisés dans le processus de fabrication, du risque de contamination accidentelle ainsi que des nombreux processus de fabrication qui peuvent tous influer sur la qualité du produit. Aussi est-il essentiel de mettre en place des mesures de contrôle de la qualité aux différentes étapes du processus de fabrication pour s'assurer que l'évaluation des risques n'est pas reportée à l'étape finale de mise en circulation des lots, laquelle ne permet pas toujours de détecter tous les changements biologiquement pertinents dans la qualité du produit. De plus, la surveillance du processus de fabrication atténue le risque que des lots soient rejetés et qu'il y ait pénurie sur le marché. Il est essentiel d'établir des spécifications appropriées pour s'assurer que les caractéristiques du produit sont maintenues pendant tout le cycle de vie du produit. II importe également de souligner que les normes de qualité de produits semblables mis au point séparément peuvent différer, pourvu que ces normes correspondent aux critères de qualité des lots s'étant révélés efficaces et sans danger lors des essais cliniques. En conclusion, une connaissance des exigences réglementaires et le maintien de discussions avec les organismes nationaux de réglementation durant chacune des étapes du développement aideront à éviter les retards inutiles et les revers potentiellement coûteux pour les fabricants. 


\section{Production de vaccins glycoconjugués par l'industrie}

\section{$D^{r}$ Richard Kensinger, Dr Don Gerson et Pradip Ghate}

Des représentants de l'industrie (Inventprise, PnuVax et Sanofi) ont expliqué en détail les bonnes pratiques de fabrication mises en place dans leurs installations, précisant que leur capacité de production était d'une échelle répondant tout au moins aux exigences liées à la fabrication d'un vaccin contre $\mathrm{Hia}$, et ont indiqué que tous possédaient une vaste expérience de la production de vaccins glycoconjugués.

Outre les exigences relatives aux bonnes pratiques de fabrication, les intervenants ont souligné les aspects importants suivants qui sont souvent négligés mais qui sont pourtant essentiels durant la production de vaccins glycoconjugués :

- Définition des critères de qualité essentiels dès le départ, avant de passer à l'étape de la production.

- Caractérisation exhaustive du lot de départ.

- Surveillance continue pour s'assurer que le produit est exempt d'impuretés sous forme de polysaccharides libres.

- Évaluation de l'évolutivité des processus par une caractérisation physicochimique pour démontrer la comparabilité des produits.

- Maintien de quantités adéquates de vaccins glycoconjugués. Besoins estimatés: Phase l, 3 à $5 \mathrm{~g}$ de polysaccharides (PS); Phase II, $10 \mathrm{~g}$ PS; Phase III, 10 à $100 \mathrm{~g}$ PS, selon les exigences en matière de posologie.

Tous les représentants de l'industrie ont confirmé qu'ils étaient intéressés à participer à la production des lots de ce vaccin glycoconjugué à des fins cliniques et/ou qu'ils avaient la capacité nécessaire à cette fin.

\section{Partenariats publics-privés dans le développement de vaccins}

\section{$D^{\text {rs }}$ Mark Kane et Jason Crawford}

Le développement des vaccins à l'échelle mondiale est passé d'une "pseudo-industrie artisanale ", où les vaccins étaient fabriqués par les instituts de santé publique ou les entreprises locales des pays, à une situation où il semble que seules les grandes sociétés pharmaceutiques peuvent fabriquer un nouveau vaccin. Les organismes comme l'Alliance mondiale pour les vaccins et la vaccination ont fait certains progrès en vue de faire échec au dogme voulant que les pays en développement n'obtiennent un vaccin que si cela profite aux pays industrialisés, mais il reste encore beaucoup à faire dans ce domaine. On pourrait s'inspirer du précédent créé par le vaccin MenAfriVac pour créer un nouveau modèle de développement pour le vaccin contre Hia. Le défi, toutefois, c'est que la Fondation Gates ou l'Alliance du vaccin ne seront probablement pas intéressés à financer un vaccin destiné à des populations potentiellement faibles de pays riches (États-Unis et Canada). La solution est donc de trouver des possibilités de financement par le gouvernement fédéral. Il est également possible que les infections causée par Hia se propagent de la même manière que celles causée par Hib.

Il semble évident qu'une garantie de marché constitue une bonne stratégie pour ce type de produit. II est donc important de tenter d'établir dès le départ à qui ce vaccin serait destiné. Il est probable que ce produit aura le statut de vaccin orphelin, ce qui aura des répercussions sur le suivi des essais cliniques et nécessitera sans doute une intensification de la surveillance post-commercialisation. Le vaccin pourrait être prêt pour le marché clinique d'ici deux ans et la possibilité de prendre appui sur l'expérience acquise avec le vaccin contre Hib sera extrêmement utile. II est par ailleurs impératif que le vaccin contre Hia soit présenté aux organismes autochtones nationaux afin d'établir des partenariats et d'élaborer avec eux des stratégies pour confirmer leurs besoins et illustrer le potentiel de ce produit. II serait très utile de mettre en place un groupe de travail pour officialiser les efforts. Cela inclurait l'élaboration d'un plan d'activités pour démontrer qu'il y aurait un « avantage » à rendre le produit disponible. Ce plan ne doit pas nécessairement indiquer une rentabilité pour le fabricant, mais doit illustrer les avantages qui en résulteraient pour "l' investisseur » et, bien sûr, pour les personnes à qui le vaccin serait destiné. Il serait également essentiel de réaliser une étude de modélisation sur le rapport coût-efficacité et sur les répercussions d'un tel vaccin. II s'impose aussi de mieux définir le fardeau de la maladie.

Les principaux points à considérer en ce qui a trait à l'épidémiologie, aux aspects économiques et au développement du vaccin se résument donc comme suit :

- Avons-nous suffisamment de données épidémiologiques sur le fardeau de la maladie chez les peuples autochtones et chez les Alaskiens d'origine?

- Avons-nous suffisamment de données des États-Unis, du Canada et le reste du monde? Comment pouvons-nous encourager la collaboration pour recueillir davantage de données à l'échelle mondiale?

- Avons-nous des données préliminaires sur le rapport coût-efficacité? Qui réalisera l'analyse de modélisation?

- Avons-nous élaboré une stratégie pour décider du choix du vecteur protéique à utiliser? Envisagerions-nous deux vaccins glycoconjugués avec différents vecteurs protéiques, un pour le Canada et l'autre pour l'Alaska?

\section{Recommandations et prochaines étapes}

L'atelier s'est conclu par une table ronde où tous les participants ont discuté des questions soulevées durant les exposés ainsi que des principales composantes sur lesquelles devront être axés les efforts futurs afin que le développement de ce vaccin potentiel puisse progresser efficacement.

Voici une liste des recommandations formulées durant l'atelier :

1. Terminer les études précliniques :

- Le choix du vecteur protéique devra être évalué avec soin, l'expérience de l'Alaska ayant démontré l'importance du vecteur pour conférer une immunité protectrice chez les nourrissons de moins de six mois.

- $\quad$ Il importe de déterminer la capacité des adjuvants approuvés chez l'humain (p. ex. l'alun) de produire des titres protecteurs.

- Il est nécessaire d'obtenir l'autorisation réglementaire nécessaire pour mener des essais bactéricides avec du sérum humain, afin de confirmer que le niveau de protection observé lors des essais cliniques correspond à celui obtenu en laboratoire. 
2. Améliorer la surveillance épidémiologique pour mieux comprendre l'étendue de la maladie dans le reste de I'Amérique du Nord et à l'échelle mondiale :

- Collaborer avec des partenaires à l'échelle nationale et internationale (p. ex. l'Organisation panaméricaine de la santé) pour mieux définir l'épidémiologie mondiale de Hia.

3. Établir des mécanismes de participation avec les organismes autochtones nationaux :

- Examiner des mécanismes visant à obtenir une participation adéquate de l'Assemblée des Premières Nations, de l'Inuit Tapiriit Kanatami et d'autres groupes de défense des intérêts, pour s'assurer que les groupes autochtones participent au processus, à la définition des orientations ainsi qu'à la prise de décisions.

4. Examiner le financement du projet en vue de produire des lots de vaccins pour la conduite des essais cliniques:

- Préparer un plan d'activités ou un document d'orientation pour démontrer la valeur d'un vaccin contre Hia.

- Démontrer le fardeau économique de la maladie et procéder à des simulations de modélisation pour illustrer le rapport coût-efficacité d'un vaccin contre Hia.

- Mettre officiellement sur pied un groupe de travail pour coordonner les activités.

\section{Conclusion}

Le nombre de cas déclarés d'infections causée par Hib a considérablement diminué après l'introduction du vaccin contre Hib au milieu des années 1980. Depuis la fin des années 1990, nous observons une augmentation concomitante des infections causée par Hia dans les communautés autochtones de l'Alaska et du nord du Canada. Des enfants et des enfants des communautés arctiques d'Amérique du Nord souffrent et meurent encore d'une infection qui semble évitable. Tous les éléments sont maintenant réunis pour le développement d'un vaccin contre Hia.

\section{Conflit d'intérêts}

A.D.C., M.G.B. et R.S.W.T. n'ont aucun conflit d'intérêts à déclarer.

M.U. bénéficie d'une subvention de recherche libre octroyée par Pfizer pour une étude indépendante. L.B. est conseiller scientifique principal auprès de Inventprise/InventVac, Redmond (État de Washington)/Vancouver (Colombie-Britannique), et conseiller stratégique auprès NÉOMED-LABS, de Montréal (Québec). Il a travaillé au sein du groupe Vaccins et immunothérapeutiques (Thérapeutiques en santé humaine) du Conseil national de recherches du Canada (CNRC) de 2011 à 2015, et agi à titre de consultant auprès du CNRC pour la mise au point d'un vaccin contre Hia. II a travaillé avec Sanofi Pasteur de 1988 à 2010 à la mise au point d'un vaccin combiné contre Hib.

\section{Collaborateurs}

Kim Barker, ministère de la Santé, gouvernement du Nunavut, lqaluit (Nunavut)

Luis Barreto, Conseil national de recherches du Canada, Ottawa (Ontario)

Andrée-Anne Boisvert, Hôpital de Montréal pour enfants, Montréal (Québec)

Simon Brascoupé, Université Carleton, Ottawa (Ontario)

Michael G. Bruce, US Centers for Disease Control and Prevention (CDC), Arctic Investigations Program, Anchorage, Alaska, États-Unis

Cindi Corbett, Agence de la santé publique du Canada, Winnipeg (Manitoba)

Andrew D. Cox, Conseil national de recherches du Canada, Ottawa (Ontario)

Jason Crawford, Centre for Drug Research and Development, Vancouver (Colombie-Britannique)

Don Gerson, PnuVax, Montréal (Québec)

Pradip Ghate, Inventprise, Seattle, État de Washington, États-Unis

Scott Halperin, Université Dalhousie, Halifax (Nouvelle-Écosse)

Mark Kane, Inventprise, Seattle, État de Washington, États-Unis

Richard Kensinger, Sanofi, Swiftwater, Pennsylvanie, États-Unis

Marlene Larocque, Assemblée des Premières Nations, Ottawa (Ontario)

Isabelle Létourneau, Instituts de recherche en santé du Canada, Montréal (Québec)

Kathleen Lydon-Hassen, Santé Canada, Ottawa (Ontario)

David Scheifele, Université de la Colombie-Britannique, Vancouver (Colombie-Britannique)

Richard Siggers, Santé Canada, Ottawa (Ontario)

Raymond Tsang, Agence de la santé publique du Canada, Winnipeg (Manitoba)

Marina Ulanova, École de médecine du Nord de l'Ontario, Thunder Bay (Ontario)

\section{Remerciements}

Nous tenons à remercier tous les participants à l'atelier pour leur collaboration et leur intérêt, ainsi que pour le temps qu'ils y ont consacré.

\section{Financement}

Cette rencontre a été organisée par le Conseil national de recherches du Canada, I'Agence de la santé publique du Canada et les Instituts de recherche en santé du Canada, sous les auspices du Conseil national de recherches du Canada. Les IRSC ont assumé les frais de déplacement de certains participants universitaires et le CNRC a offert des rafraîchissements durant les pauses et assumé les frais de déplacement de l'animateur de la conférence. 


\section{Références}

1. Gouvernement du Canada. Guide canadien d'immunisation : Partie 4 - Agents d'immunisation active, Vaccin contre Haemophilus Influenzae de type B [Internet]. Ottawa: ASPC [mise à jour 2017 mars; consulté le 24 mars 2017]. Disponible à l'adresse : https://www.canada.ca/fr/santepublique/services/publications/vie-saine/guide-canadienimmunisation-partie-4-agents-immunisation-active/page-5vaccin-contre-haemophilus-influenzae-type-b.html.

2. Boisvert AA, Moore D. Invasive disease due to Haemophilus influenzae type A in children in Canada's north: A priority for prevention. Can J Infect Dis Med Microbiol. 2015;26:291-292.

3. Tsang RS, Li YA, Mullen A, Baikie M, Whyte K, Shuel M, Tyrrel G, Rotondo JA, Desai S, Spika J. Laboratory characterization of invasive Haemophilus influenzae isolates from Nunavut, Canada, 2000-2012. Int J Circumpolar Health 2016;75:29798.

4. PubMLST [Internet]. Haemophilus influenzae MLST Databases. [consulté le 24 mars 2017]. Disponible à l'adresse : http://pubmlst.org/hinfluenzae/.

5. Bruce MG, Zulz T, DeByle C, Singleton R, Hurlburt D, Bruden D, Rudolph K, Hennessy T, Klejka J, Wenger JD. Invasive disease caused by Haemophilus influenzae serotype $a$, an emerging pathogen in Alaska. Emerg Infect Dis. 2013;19:932-937.

6. Bender JM, Cox CM, Motttice S, She RC, Korgenski K, Daly JA. Invasive Haemophilus influenzae disease in Utah children: An 11-year population-based study in the era of conjugate vaccine. Clin Infect Dis. 2010;50:e41-e46.

7. Rubach MR, Bender JM, Mattice S, Hanson K, Weng HYC, Korgenski K, Daly JA, Pavia AT. Increasing incidence of invasive Haemophilus influenzae disease in adults, Utah, USA. Emerg Infect Dis. 2011;17:1645-1650.

8. Zanelle RC, Casagrande ST, Bokermann S, Almeida SCG, Brandileone MCC. Characterization of Haemophilus influenzae isolated from invasive disease in Brazil from 1990 to 1999. Microbial Drug Resistance. 2002;8: 67-72.

9. Ribeiro GS, Lima JBT, Reis JN, Gouveia EL, Cordeiro SM, Lobo TS, Pinheiro RM, Ribeiro CT, Neves AB, Salgado K, Silva HR, Reis MG, Ko Al. Haemophilus influenzae meningitidis 5 years after introduction of the Haemophilus influenzae type b conjugate vaccine in Brazil. Vaccine. 2007; 25:4420-4428

10. Scheifele DW, Halperin SA, and members of the Health Canada/CPS Immunization Monitoring Program Active (IMPACT). A model of active surveillance of vaccine safety. Semin Pediatr Infect Dis. 2003;14:213-219.

11. Bettinger JA. Halperin SA, Vaudry W, Law BJ, Scheifele DW. Le programme canadien de surveillance active de
I'immunisation (IMPACT) : Surveillance active des effets secondaires suivant l'immunisation et des maladies évitables par la vaccination. Relevé des maladies transmissible au Canada. 2014;40(S-3). Disponible à l'adresse : http://www. phac-aspc.gc.ca/publicat/ccdr-rmtc/14vol40/dr-rm40s-3/ comment-d-fra.php.

12. Ward J, Brenneman G, Letson GW, Heyward WL, Alaska $\mathrm{H}$. Influenzae Vaccine Study Group. Limited efficacy of a Haemophilus influenzae type b conjugate vaccine in Alaska native infants. New Eng J Med. 1990;323:1393-1401.

13. Singleton R, Hammitt L, Hennessy T, Bulkow L, DeByle C, Parkinson A, Cottle TE, Peters H, Butler JC. The Alaska Haemophilus influenzae Type b Experience: Lessons in controlling a vaccine-preventable disease. Pediatrics. 2006;118:421-429.

14. Bulkow LR, Wainwright RB, Letson GW, Chang WJ, Ward JI. Comparative immunogenicity of four Haemophilus influenzae type b conjugate vaccines in Alaska native infants. Pediatr Infect Dis J. 1993;12:484-492.

15. Brown VM, Madden S, Kelly L, Jamieson FB, Tsang RS, Ulanova M. Invasive Haemophilus influenzae disease caused by non-type b strains in Northwestern Ontario, Canada, 2002-2008. Clin Infect Dis. 2009;49:1240-3.

16. Kelly L, Tsang R, Morgan A, Jamieson F, Ulanova M. Invasive disease caused by Haemophilus influenzae type a in Northern Ontario First Nations communities. J Med Microbiol. 2011;60:384-390.

17. Nix EB, Williams K, Cox AD, St.-Michael F, Romero-Steiner S, Schmidt DS, McCready WG, Ulanova M. Naturally acquired antibodies against Haemophilus influenzae type a in Aboriginal adults, Canada. Emerg Infect Dis. 2015;21:273279

18. Halperin SA, Scheifele D, Duval B, et al. Canadian Association for Immunization Research and Evaluation (CAIRE) Guidelines for industry sponsored clinical trial and epidemiology contract research. Hum Vaccines. 2005;140-2.

19. McNeil SA, Andrew MK, Ye L, Haguinet F, Hatchette TF, ElSherif M, LeBlanc J, Ambrose A, McGeer A, McElhaney JE, Loeb M, MacKinnon-Cameron D, Sharma R, Dos Santos G, Shinde V. Investigators of the Serious Outcomes Surveillance Network of the Canadian Immunization Research Network (CIRN). Interim estimates of 2014/15 influenza vaccine effectiveness in preventing laboratory-confirmed influenzarelated hospitalisation from the Serious Outcomes Surveillance Network of the Canadian Immunization Research Network. Euro Surveill. 2015;20:21024.

20. Rubinstein E, Predy G, Sauvé L, Hammond GW, Aoki F, Sikora W, Li Y, Law B, Halperin S, Scheifele D. The responses of Aboriginal Canadians to adjuvanted pandemic (H1N1) 2009 influenza vaccine. CMAJ 2011;183:1033-1037. 